\title{
Comparison of theoretical and experimental dielectric functions: Electron energy-loss spectroscopy and density-functional calculations on skutterudites
}

\author{
$\varnothing$. Prytz, ${ }^{1}$ O. M. Løvvik, ${ }^{1}$ and J. Taft $\varnothing^{2}$ \\ ${ }^{1}$ University of Oslo, Centre for Materials Science and Nanotechnology, P.O.Box 1126 Blindern, N-0318 Oslo, Norway \\ ${ }^{2}$ University of Oslo, Department of Physics, P.O.Box 1048 Blindern, N-0316 Oslo, Norway
}

(Received 13 July 2006; revised manuscript received 2 October 2006; published 13 December 2006)

\begin{abstract}
We explore the possibility of combining density functional theory (DFT) and electron energy loss spectroscopy (EELS) to determine the dielectric function of materials. As model systems we use the skutterudites $\mathrm{CoP}_{3}, \mathrm{CoAs}_{3}$, and $\mathrm{CoSb}_{3}$ which are prototypes for thermoelectric materials. We achieve qualitative agreement between the theoretically and experimentally obtained low energy-loss spectra and dielectric function. Some of the remaining discrepancies may be caused by the challenge of refining the experimental spectra before Kramers-Kronig analysis. However, contrary to what is the case for some crystals with less complicated electronic structure, the DFT calculated plasmon energies deviate significantly from the experimental values. The great accuracy with which the plasmon energy can be determined by EELS, suggests that this technique may provide valuable inputs in further efforts to improve DFT calculations. The use of EELS as the experimental technique may become particularly powerful in studies of small volumes of materials.
\end{abstract}

DOI: 10.1103/PhysRevB.74.245109

PACS number(s): 71.45.Gm, 79.20.Uv, 71.20.-b

\section{INTRODUCTION}

Many electron energy-loss spectroscopy (EELS) studies have focused on the energy loss edges corresponding to the excitation of core electrons, usually occurring above $100 \mathrm{eV}$. These excitations normally involve dipole allowed transitions from an initial state which is highly localized in energy, to a final state above the Fermi level. The relative intensity of these features can be used for quantitative studies of composition, while the fine structure will reflect the local density of empty states. Numerous studies have used these core-loss edges to investigate the electronic structure and bonding of materials. ${ }^{1-7}$

In comparison, the energy-loss features appearing at lower energies (below $50 \mathrm{eV}$ ) are less studied, even though they can be orders of magnitude more intense than the core losses discussed above. These low energy-loss spectra contain a wealth of information, e.g., the dielectric function of the material can be extracted through a Kramers-Kronig analysis of high quality spectra. The energy loss processes taking place in this energy region can be divided into two main categories.

One category is the collective excitations of the valence electrons, which can be considered as a plasma. These excitations occur when the incident electron perturbs the outer valence electrons of the material, causing collective oscillations (called plasmons) with eigenfrequency $\omega_{p}$. The ideal free electron plasma frequency is fully determined by the valence electron density. These plasmons are usually the single most intense feature available in the energy-loss spectrum. However, studies of the plasmon energies reveal that the electrons in real materials do not behave exactly as a free electron plasma. This is because the electron gas oscillates in a solid with a potential set up by the atom cores. Furthermore, single electron excitations may contribute to raising or lowering the plasmon energy. In principle, careful investigation of the plasmon energy of materials could reveal important information on their electronic structure. ${ }^{8}$ Unfortunately, an interpretation of the observed spectra along these lines is extremely demanding, and in practice the plasmon energies have mainly been used to study changes in valence electron density, e.g., resulting from strain or hydration of metals. ${ }^{9,10}$

In addition to the plasmons, the low energy-loss region contains low energy interband transitions of single electrons. These interband transitions cannot be interpreted with reference to the unoccupied density of states only. The initial states of these transitions are located close to the Fermi level, and are usually not localized in energy. The energy losses must therefore be discussed in light of a convolution of initial states below the Fermi level with final states above it, reflecting a dipole selected joint density of states. Due to the dependence on both initial and final states we can expect these low energy-loss interband transitions to be very sensitive to bonding effects in the material, potentially revealing information on the electronic structure. As with the plasmon energies, the interpretation of these spectra is very challenging and has not been widely applied to study the electronic structure of materials. For this region of the energy-loss spectrum to be more extensively used to study the electronic structure, there is a need for strong interaction between theory and experiment.

However, the calculation of electronic excitations from first principles is a highly non-trivial problem, which so far has no satisfactory solution. ${ }^{11,12}$ Some methods are in principle exact, but their scaling behavior with system size prohibits their use except for the smallest model systems- the configuration-interaction method ${ }^{13}$ and Green's function based quasi-particle approaches ${ }^{14,15}$ are examples of this. Time-dependent DFT, on the other hand, is also in principle exact and has much better scaling behavior, but the correct form of the exchange-correlation function and its variation (kernel) are not known, and no good general approximations have been found so far. ${ }^{16-18}$ This is a very active field of research, and a number of possible solutions are emerging. ${ }^{19-23}$ However, these are still usually accessible only for very small systems. 
Thus, for calculating these properties for larger unit cells, one has to turn to simpler DFT response calculations, in which the Kohn-Sham eigenvalues are taken as quasiparticles from which optical excitation energies may be calculated. This is not formally justified, but direct comparisons of calculated core loss spectra with experimental results have shown good correspondence for some systems. ${ }^{24-27}$ However, the correspondence of theoretical and experimental low energy-loss spectra is less well investigated.

Recently, Keast ${ }^{28}$ compared the theoretical plasmon energy and low energy-loss spectra for a number of elemental solids and some binary alloys with experiments with good results. Also, Moreau and Cheynet ${ }^{29}$ compared band structure calculations with EELS experiments on BN filaments. Furthermore, a satisfactory agreement between experimental and calculated dielectric function has already been achieved for simple crystals such as $\mathrm{Si}^{30}$

We intend to approach more complicated materials than the tetrahedrally coordinated semiconductors: crystals with a larger unit cell, without a well-defined number of valence electrons and more than one type of nearest neighbor bonds. At the same time we look for a material system without stoichiometry problems and crystals of high quality. Interesting candidates are materials with the skutterudite structure. These are line compounds, and virtually defect free as judged by transmission electron microscopy. In addition, materials with this structure are highly interesting thermoelectric materials which strengthen their candidacy as excellent model systems.

We study the binary skutterudites $\mathrm{CoP}_{3}, \mathrm{CoAs}_{3}$, and $\mathrm{CoSb}_{3}$. The crystal structure of these materials is cubic. Co has six $\mathrm{P}$ nearest neighbors $\left(d^{2} s p^{3}\right.$ hybrid bonds), while the pnicogen is tetrahedrally coordinated with two Co and two $\mathrm{P}$ nearest neighbors ( $s p^{3}$ hybrid bonds). ${ }^{31}$ Several band structure calculations have been performed on these and similar materials using DFT. ${ }^{32-34}$ However, apart from measurements of the electronic conductivity and Seebeck coefficient, there are few direct comparisons between calculations and experimental results for these materials, particularly when it comes to detailed information about their electronic structure. ${ }^{35-38}$

The aim of the present work is to compare DFT calculated and EELS extracted dielectric functions for crystals with more complex bonding than has previously been approached. We believe that this combination of experimental and theoretical methods to study the electronic structure may become a powerful synergistic approach towards understanding the electronic properties of materials. A major motivation for using EELS is that this technique may become particularly powerful for nanomaterials, and when only small amounts of materials are available, since EELS spectra can be acquired from nanometer sized regions.

\section{EXPERIMENTAL PROCEDURES, DATA ANALYSIS, AND THEORETICAL METHODS}

\section{A. Synthesis and sample preparation}

The skutterudite samples were prepared by direct reaction of the constituent elements during heating in evacuated and sealed silica tubes. The homogeneity of the samples were subsequently checked by $\mathrm{x}$-ray powder diffraction in transmission with a Siemens D5000 diffractometer using monochromated $\mathrm{Cu}-K \alpha_{1}$ radiation. The samples were found to be almost completely single phase, with only small amounts of impurity phases present. The lattice constant $a$ and position parameters $y$ and $z$ were refined using the General Structure Analysis System (GSAS). ${ }^{39}$ The experimental values of this study show good agreement with those reported earlier. ${ }^{40-42}$ Samples for transmission electron microscopy (TEM) and electron energy loss spectroscopy (EELS) were prepared by grinding in ethanol in an agate mortar and deposited on a carbon film suspended on a copper mesh. The composition of the samples was then checked using energy dispersive $\mathrm{X}$-ray analysis (EDS) in a JEOL 2000FX TEM operated at $200 \mathrm{kV}$ fitted with a Tracor Northern EDS detector and a Noran System Six analysis system. The EDS analyses showed that the samples were of the correct composition within the experimental accuracy. Furthermore, electron diffraction and imaging in TEM showed that the crystals were of excellent quality.

\section{B. Electron energy loss spectroscopy}

The EELS studies were performed using a post column Gatan imaging filter fitted to a field emission JEOL 2010F TEM operated at $197 \mathrm{kV}$. The TEM was operated in image mode with a collection semiangle $\beta=6 \mathrm{mrad}$, and a slightly convergent electron beam with incidence semiangle $\alpha$ approximately $3 \mathrm{mrad}$.

The spectra were obtained with an energy resolution of $1.2 \mathrm{eV}$, as determined by the full width at half maximum of the zero-loss peak, and a spectrometer dispersion of $0.2 \mathrm{eV} /$ channel. The energy scale was further calibrated using the plasmon peak of pure $\mathrm{Si}$ as a standard. The spectra were corrected for channel-to-channel gain variations in the detector, and the dark current was subtracted. The spectra were obtained from sample areas of thickness between 1 and 2 times the mean free path of the fast electrons. This ensured that the relative contribution of the surface plasmons was negligible.

Figure 1 shows an example of the spectra obtained in this fashion, the multiple plasmon peaks appear because an incident electron may lose energy to plasmon excitation more than once. The effects of multiple scattering were removed through a Fourier-log deconvolution as implemented in the Gatan EELS Analysis Software (see Egerton ${ }^{43}$ for details). Thus the single scattering distribution $S(E)$ was obtained.

The subtraction of the broad tails of the zero loss peak is of special importance when studying the low energy-loss area of the spectrum. A zero loss profile obtained without the sample was used in the deconvolution of each spectrum to identify the elastic contribution. This premeasured profile was fitted to the spectra in the region $1.5-2.5 \mathrm{eV}$. As a result of this fitting, spectral information in this area may be lost. Figure 2 shows an example of how this fitting was done.

The energy loss suffered by an incident electron is determined by the electronic response of the material, which is described by the complex dielectric function $\varepsilon(E)$. The di- 


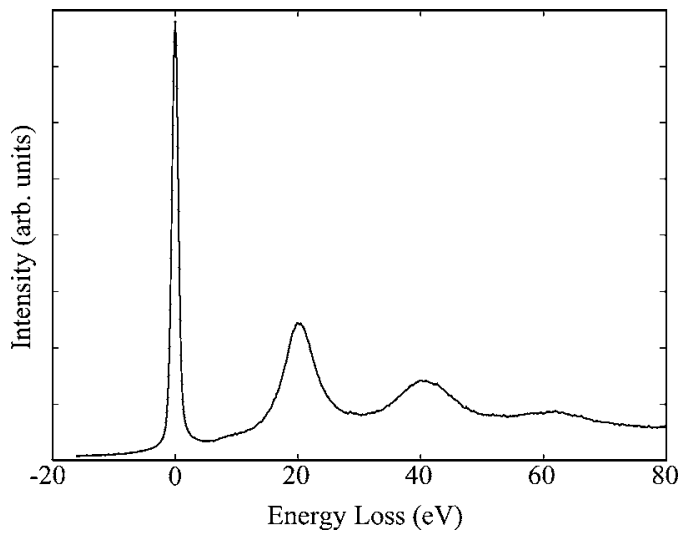

FIG. 1. In the raw data obtained from the EELS experiment (before deconvolution), multiple plasmons are observed. Here is shown an unprocessed experimental spectrum from $\mathrm{CoAs}_{3}$ where we observe a plasmon energy of slightly more than $20 \mathrm{eV}$.

electric function is related to the experimental single scattering distribution through

$$
S(E) \propto \operatorname{Im}\left(-\frac{1}{\varepsilon(E)}\right)=\frac{\varepsilon_{2}}{\varepsilon_{1}^{2}+\varepsilon_{2}^{2}} .
$$

The full dielectric function can be retrieved from the experimental spectrum through a Kramers-Kronig analysis. This was also done using the Gatan EELS Analysis software, see Egerton ${ }^{43}$ for details.

\section{Density functional theory}

The calculations were performed using density functional theory at the generalized gradient approximation level. Structural relaxations and densities of states (DOS) were calculated using the Vienna ab initio Simulation Package (VASP). ${ }^{44,45}$ For VASP, the energy cutoff for the plane wave expansion was $650 \mathrm{eV}$, the $\mathbf{k}$-point distances were less than $0.20 \AA^{-1}$, the criterion for self-consistency was energy convergence within $10^{-5} \mathrm{eV}$, and spin polarization was allowed. Ionic relaxation was said to be achieved when all forces were less than $0.05 \AA^{-1}$.

The dielectric function of the skutterudites was obtained from the Optic package of the wIEN2K code. ${ }^{46}$ In these calculations, the dipole approximation is assumed valid, and only transitions satisfying the dipole selection rule $\Delta l= \pm 1$ are considered. Convergence of the WIEN $2 K$ results were achieved with the product $R_{\mathrm{MT}} K_{\max }=7$, the largest vector in the charge density Fourier expansion $G_{\max }=14$, and the number of $\mathbf{k}$ points chosen to be 1000 .

The calculated dielectric function can be compared to the experimental dielectric function obtained as described above. Alternatively, a theoretical spectrum can be calculated according to Eq. (1) and compared directly to the experimental results. The theoretical plasmon energy of the three compounds was obtained from the maximum of this theoretical spectrum.

\section{RESULTS AND DISCUSSION}

The cell parameters and positional parameters $y$ and $z$ obtained from the fully relaxed cell of the DFT calculations

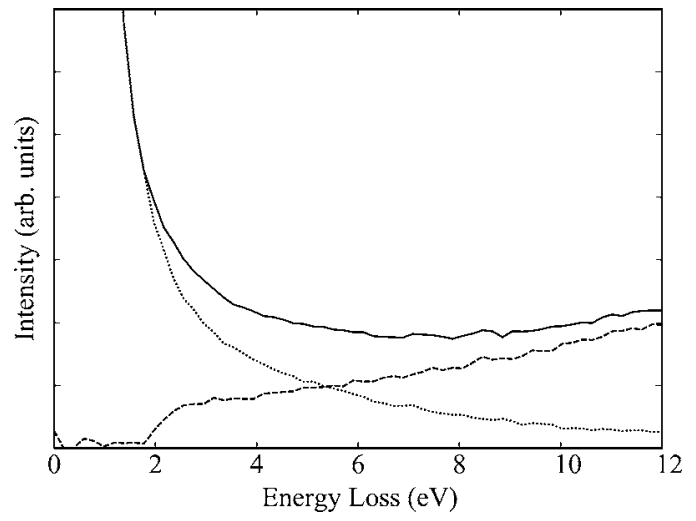

FIG. 2. Example of the zero-loss peak removal and Fourier-log deconvolution. The unprocessed raw data is shown with a fully drawn line, while the fitted zero-loss peak is the dotted line. The resulting spectrum after subtraction of the zero loss peak and deconvolution is shown with a dashed line.

are listed in Table I along with previously reported XRD values and those obtained in this study. The overall agreement between theory and experiments is very good, although the DFT calculations seem to slightly overestimate the cell size (less than $1 \%$ ).

The total density of states (DOS) and the symmetry projected local density of states for the three skutterudites are shown in Fig. 3. In general, the DOS for the three compounds show great similarity. The Co $d$ states are split into states of $t_{2 g}$ and $e_{g}$ symmetry due to the octahedral environment of the Co atoms, with the majority of the Co $d$ states strongly peaked in an area approximately $2-2.5 \mathrm{eV}$ below the Fermi level. The pnicogen $p$ states are rather uniformly distributed above and below the Fermi level, hybridizing with the Co $d$ states and contributing heavily to the total DOS in the -7 to $+5 \mathrm{eV}$ region. The Co $s$ and $p$ states contribute only to a small degree to the total DOS, while the pnicogen $s$ states dominate in the region $9-15 \mathrm{eV}$ below the Fermi level. In $\mathrm{CoP}_{3}$, the Fermi level is located at the very lower edge of the conduction band, while in $\mathrm{CoAs}_{3}$ and $\mathrm{CoSb}_{3}$ it is shifted slightly downward towards the valence band, into the pseudogap where the density of states is very small. It is also evident that this pseudogap is largest for $\mathrm{CoP}_{3}$, and decreases in width in the skutterudites with the heavier pnicogens and larger unit cells. These results are in good agreement with previous work ${ }^{32,34,35}$ and will later be used in the interpretation of experimental and theoretical spectra obtained in this study.

\section{A. Plasmon excitations}

The experimental energy-loss spectra from the skutterudites show rather sharp plasmon peaks, and pronounced additional peaks associated with multiple plasmon losses are visible as exemplified in Fig. 1. This behavior is very similar to that displayed by semiconductors and simple metals, while transition metals and their oxides usually display only a single broad plasmon. The Fourier-log deconvolution successfully removed the contributions from multiple scattering, giving the single scattering distributions shown in Fig. 4(a). 
TABLE I. The lattice constant $a$, and position parameters $y$ and $z$ for the three skutterudites obtained by $\mathrm{XRD}$ and DFT. Previously reported experimental values are listed for comparison. The DFT cell parameter for $\mathrm{CoP}_{3}$ is from a previous study (Ref. 47).

\begin{tabular}{llll}
\hline \hline & \multicolumn{1}{c}{$a(\AA)$} & \multicolumn{1}{c}{$y$} & \multicolumn{1}{c}{$z$} \\
\hline $\mathrm{CoP}_{3}$ & & & \\
$\quad \mathrm{XRD}$ (this work) & $7.7163(1)$ & $0.34812(3)$ & $0.14285(29)$ \\
$\quad$ Previously reported XRD (Ref. 40) & $7.7112(8)$ & $0.34895(6)$ & $0.14513(6)$ \\
$\quad$ Previously reported DFT (Ref. 47) & 7.735 & 0.348 & 0.146 \\
$\mathrm{CoAs}_{3}$ & & & \\
$\mathrm{XRD}$ (this work) & $8.2056(2)$ & $0.3384(3)$ & $0.1481(25)$ \\
Previously reported XRD (Ref. 41) & $8.195(3)$ & $0.3431(1)$ & $0.1503(1)$ \\
$\mathrm{DFT}$ (this work) & 8.277 & 0.341 & 0.152 \\
$\mathrm{CoSb}_{3}$ & & & \\
$\mathrm{XRD}$ (this work) & $9.0463(6)$ & $0.3299(6)$ & $0.1560(6)$ \\
Previously reported XRD (Ref. 42) & $9.0385(3)$ & $0.33537(4)$ & $0.15788(4)$ \\
DFT (this work) & 9.117 & 0.334 & 0.160 \\
\hline
\end{tabular}

We immediately recognize the intense plasmon peaks associated with collective oscillations of the valence electrons. Also visible are energy loss features associated with the $M_{4,5}$ and $N_{4,5}$ excitations of outer core electrons of As and $\mathrm{Sb}$, respectively.

The theoretically calculated spectra for the compounds are shown in Fig. 4(b). Similar to the experimental results, these show a single sharp plasmon peak, and loss features on the high energy side of the plasmon peak associated with the excitation of outer core electrons. The experimental plasmon peaks are significantly broader than those of the theoretical spectra, a feature that can be explained by broadening caused by the limited energy resolution of the experiments. The experimentally obtained plasmon energies are given in Table II, alongside theoretical values obtained from the DFT calculations.

The plasmon energy of materials displaying narrow, well defined plasmon peaks is often successfully predicted by the

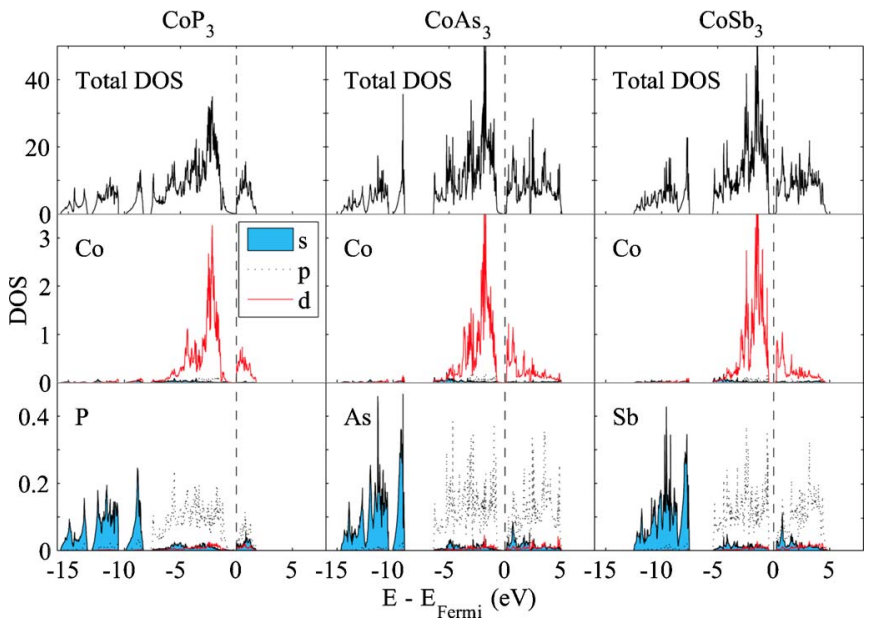

FIG. 3. (Color online) Total and local projected density of states for $\mathrm{CoP}_{3}, \mathrm{CoAs}_{3}$, and $\mathrm{CoSb}_{3}$. The total density of states for $\mathrm{CoP}_{3}$ was published in a previous study (Ref. 47). free electron Drude model. In this model, the plasmon energy is given by $E_{p}^{D}=\hbar \omega_{p}^{D}$, where $\omega_{p}^{D}$ is the frequency of the plasma oscillation given by ${ }^{8}$

$$
\omega_{p}^{D}=\sqrt{\frac{n e^{2}}{\varepsilon_{0} m_{0}}} .
$$
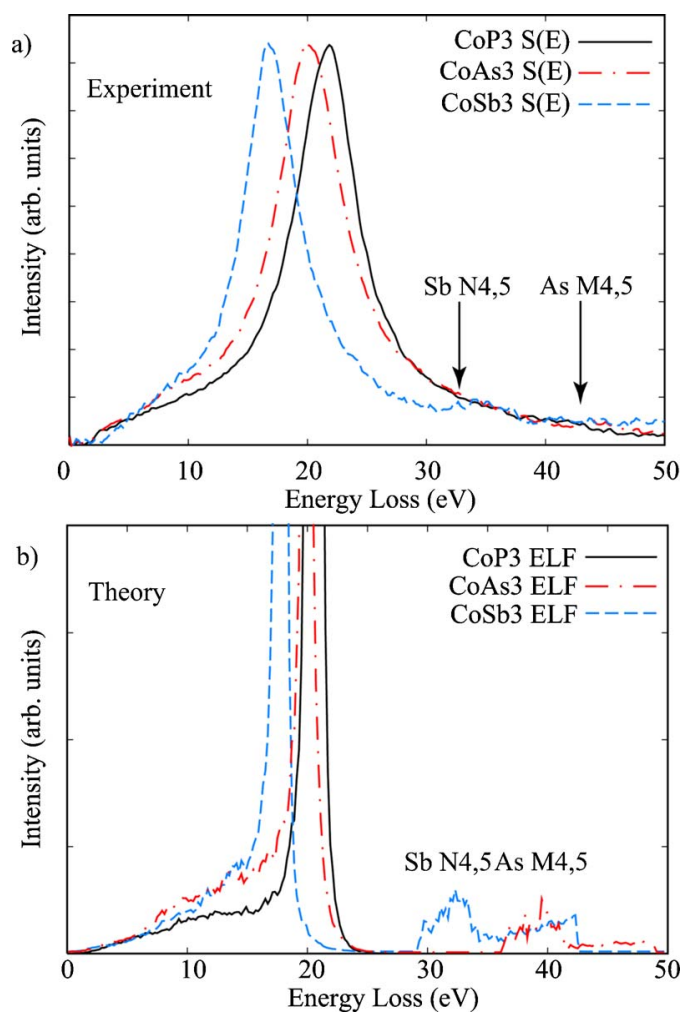

FIG. 4. (Color online) Experimental and theoretical low energyloss spectra for the three skutterudites. (a) Experimental spectra showing a single plasmon peak. (b) Theoretical spectra. The energy loss peaks associated with the As $M_{4,5}$ transitions and the $\mathrm{Sb} N_{4,5}$ transitions are visible in both the experimental and theoretical spectra. 
TABLE II. Experimental plasmon energy $E_{p}^{\exp }$ for the three skutterudites. We also list the plasmon energy calculated from the Drude model $E_{p}^{D}$ and the plasmon energy found from the ELF based on the dielectric function $E_{p}^{\mathrm{DFT}}$. The relative deviation $(\Delta)$ of the calculated vs the experimental energy is also listed.

\begin{tabular}{lccccc}
\hline \hline & $E_{p}^{\exp } / \mathrm{eV}$ & $E_{p}^{D} / \mathrm{eV}$ & $\Delta$ & $E_{p}^{\mathrm{DFT}} / \mathrm{eV}$ & $\Delta$ \\
\hline $\mathrm{CoP}_{3}$ & $21.67 \pm 0.08$ & 20.78 & $-4.1 \%$ & 20.9 & $-3.55 \%$ \\
$\mathrm{CoAs}_{3}$ & $20.34 \pm 0.11$ & 18.95 & $-6.8 \%$ & 19.9 & $-2.16 \%$ \\
$\mathrm{CoSb}_{3}$ & $16.88 \pm 0.09$ & 16.37 & $-3.0 \%$ & 17.9 & $+6.04 \%$ \\
\hline \hline
\end{tabular}

Here, $n$ is the density of valence electrons, $e$ and $\varepsilon_{0}$ are the elementary electric charge and the permittivity of free space, while $m_{0}$ is the free electron mass. Table II also lists the plasmon energy found by using Eq. (2) and assuming three valence electrons for Co and five electrons for P, As, and $\mathrm{Sb}$, giving a total of 144 valence electron per unit cell for all the skutterudites

Using this valence electron count, the Drude model predicts a reduction of $E_{p}$ as the unit cell size increases and the valence electron density decreases. We see that this is in fact the case, with $\mathrm{CoSb}_{3}$ having the largest unit cell and the lowest plasmon energy, while $\mathrm{CoP}_{3}$ has the smallest cell size and thereby the largest plasmon energy. Other than this, we note that the Drude model significantly, and consistently, underestimates the plasmon energy of the skutterudites. The deviation between experimental and predicted plasmon energies ranges from 4 to nearly $7 \%$.

The basic premise of the Drude model is that the valence electrons can be considered as simple harmonic oscillators. The plasmon frequency $\omega_{p}^{D}$ is then identified as the frequency of resonant oscillation. However, when transition metals are involved, this simple picture is complicated by the fact that these elements have an outer shell consisting of a large number of $d$ electrons that are bound to a varying degree. Thus, the transition metals and their oxides usually display a single, broad plasmon peak, and the Drude model often fails to predict their plasmon energies. In comparison, simple metals such as $\mathrm{Be}, \mathrm{B}, \mathrm{Na}$, and $\mathrm{Al}$, and semiconductors such as $\mathrm{Si}, \mathrm{Ge}$, and GaAs have sharp plasmon peaks near the predicted value, and peaks associated with the multiple losses are readily observed. ${ }^{8,43,48}$ The skutterudites represent a different class of materials where the plasmons display the characteristics of simple metals or semiconductors, but the Drude model fails to predict their energy.

When turning to the DFT calculations, we note that the correspondence with the experimental plasmon energies is not much better than for the Drude model; the plasmon energy of $\mathrm{CoSb}_{3}$ is significantly overestimated (6\%) while those of $\mathrm{CoP}_{3}$ and $\mathrm{CoAs}_{3}$ are underestimated by $2-4 \%$.

Despite this, the results presented here are only slightly poorer than that achieved for simpler systems, ${ }^{28}$ and for $\mathrm{Si}$ using more sophisticated methods. ${ }^{30}$ We have also performed spin-polarized calculations (not presented), but these give an even worse result both in the position of the plasmon peak and the line shape relative to the experimental ones. One possible reason may be a fortuitous cancellation of errors in the spin-restricted calculations. To improve these numbers further, more sophisticated methods than standard DFT must be used, such as time-dependent DFT or Green's function based approaches (such as Hedin's $G W$ method $^{49}$ ) including electron-hole interactions - this is beyond the scope of the current work.

Thus, the main advantage of the DFT calculations, compared to the free electron model, is that also the line shapes of the absorption spectra, as well as the dielectric function, are relatively easily achieved. This is due to the former being based on the calculated band structure of the compounds.

\section{B. Single electron transitions in the low energy-loss region and the dielectric function}

The intensity of the EEL spectrum associated with single electron transitions is given by ${ }^{50}$

$$
\begin{aligned}
I \propto \mid & \mid\left\langle\Psi_{f}\left|e^{i \Delta \mathbf{k} \cdot \mathbf{r}}\right| \Psi_{i}\right\rangle^{2} \\
& =\left|\left\langle\Psi_{f}\left|1+i \Delta \mathbf{k} \cdot \mathbf{r}+\frac{(i \Delta \mathbf{k} \cdot \mathbf{r})^{2}}{2 !}+\cdots\right| \Psi_{i}\right\rangle\right|^{2} \\
& \approx \mid\left\langle\Psi_{f}|i \Delta \mathbf{k} \cdot \mathbf{r}| \Psi_{i}\right\rangle^{2} .
\end{aligned}
$$

Here $\Psi_{i}$ and $\Psi_{f}$ designate the occupied initial and unoccupied final states, $\Delta \mathbf{k}$ is the momentum transfer from the incident electron to the excited electron, while $\mathbf{r}$ is the position of the electron with the atom core as the origin. If the product $\Delta \mathbf{k} \cdot \mathbf{r}$ is sufficiently small, the higher powers of the Taylor series in Eq. (3) vanish, and the dipole approximation is to be considered valid. All observed transitions then obey the dipole selection rule $\Delta l= \pm 1$. This situation is usually achieved by using a small entrance aperture for the spectrometer, which limits the scattering vector $\Delta \mathbf{k}$.

In core-loss spectroscopy, the initial states are atomic in nature and are usually assumed to be highly localized in energy. The spectral features are therefore mainly due to variations in the density of empty states above the Fermi level. However, we wish to examine the fine structure of the low energy-loss region below the plasmons. In these experiments, both the initial and final states are located close to the Fermi level and distributed in energy. The observed energy loss features are then related to a joint density of states. We expect this fine structure to be very sensitive to the bonding in the skutterudites. In principle, it is also possible to measure the band-gap of materials using EELS. However, this is practical only for wide band-gap semiconductors and insulators using systems with sub-eV energy resolution. ${ }^{51}$

Figure 5 shows the low energy-loss region of the three skutterudites after deconvolution and with the zero loss peak removed, together with the calculated spectra. Here, theoretical spectra calculated both with and without a $0.3 \mathrm{eV}$ Lorentzian broadening are shown. At the high energy side of these figures, we see the rising intensity of energy loss due to the plasmon excitations. The plasmon peaks extend outside the figures.

On the low energy side of the plasmon peaks, there are trailing shoulders in both the theoretical and experimental spectra. These shoulders are associated with the low energy single electron transitions close to the Fermi level. We note that for all three materials this shoulder is rather featureless, 

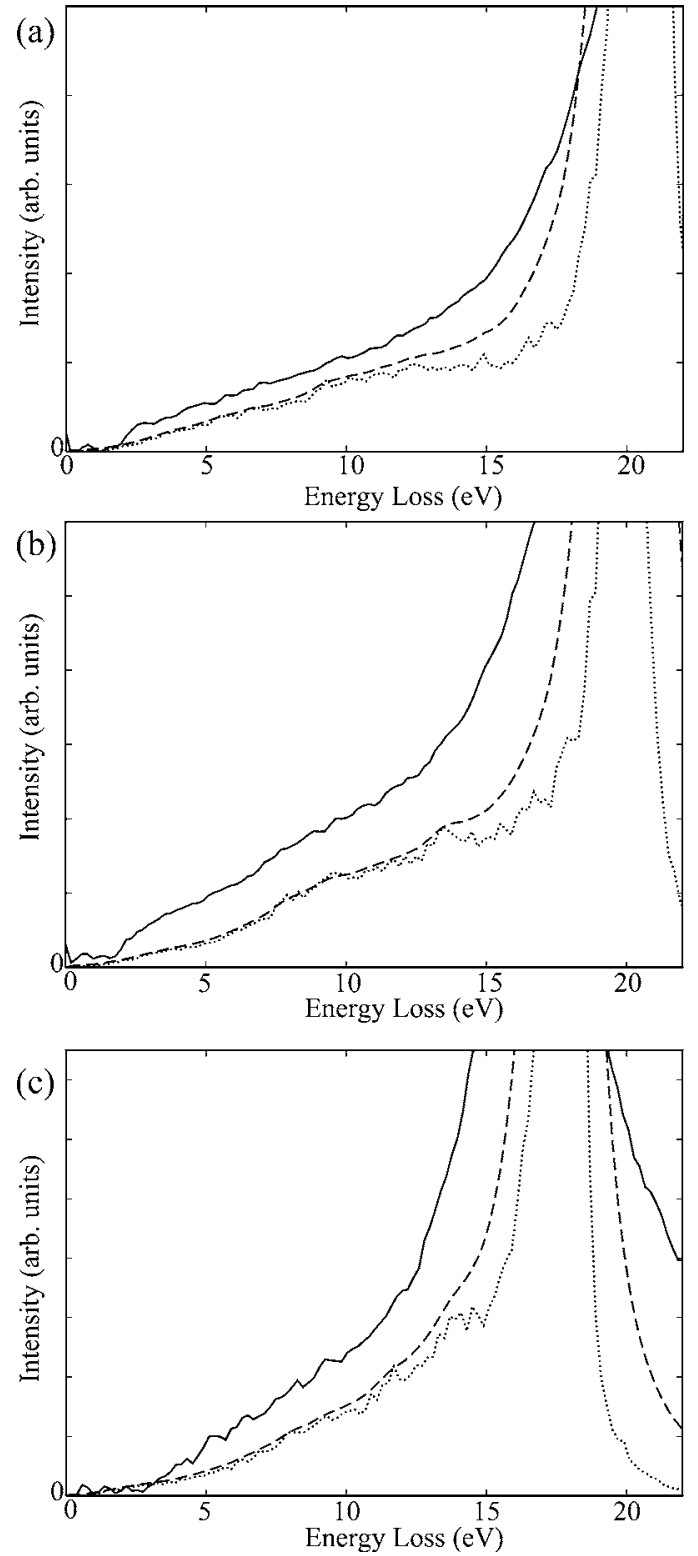

FIG. 5. The experimental (fully drawn line), and theoretical energy-loss spectra of (a) $\mathrm{CoP}_{3}$, (b) $\mathrm{CoAs}_{3}$, and (c) $\mathrm{CoSb}_{3}$. The theoretical spectra have been calculated with (dashed) and without (dotted) a $0.3 \mathrm{eV}$ Lorentzian broadening. The relative scaling of the theoretical data with respect to the experimental data is arbitrary, no direct comparison of the absolute intensity is possible.

reflecting the uniform unoccupied density observed in Fig. 3. Regardless of this, the qualitative shape of this low energyloss region varies somewhat in the three materials, and this variation is reasonably reproduced in the calculated spectra. Furthermore, for $\mathrm{CoAs}_{3}$ it is possible to identify individual features in both the theoretical and experimental spectra. In particular, features in the energy-loss spectra just below $10 \mathrm{eV}$ and around $13 \mathrm{eV}$ could represent dipole allowed transition from the very sharp As $s$ feature located approximately $8-10 \mathrm{eV}$ below the Fermi level to available states with $p$ symmetry, see Fig. 3. Even though the details available in the experimental spectra are rather limited in comparison to the theoretical spectra, the overall correspondence
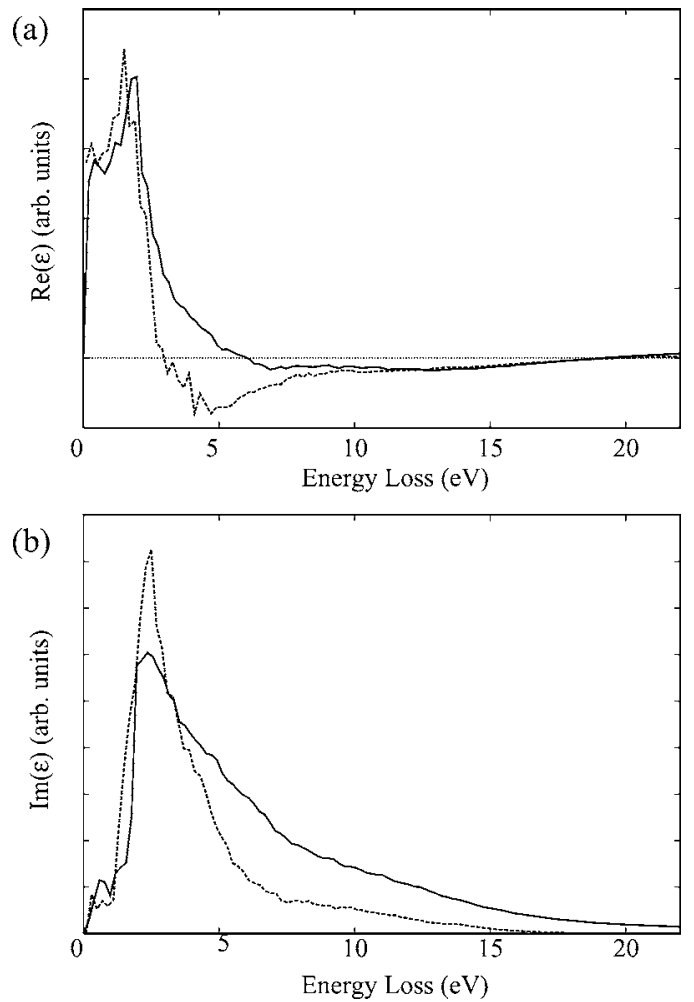

FIG. 6. (a) The real and (b) imaginary parts of the dielectric function of $\mathrm{CoAs}_{3}$. The experimentally obtained function is shown with a fully drawn line, while the theoretical function is a dashed line.

is good, indicating that the calculated electronic structure fairly accurately reflects the real bonding in the skutterudites.

At these low energies, the possible breakdown of the dipole approximation needs to be considered. As explained earlier, the DFT calculations only take into account electron transitions satisfying the dipole selection rule $\Delta l= \pm 1$. However, since we are considering transition of the outer shell electrons, the product $\Delta \mathbf{k} \cdot \mathbf{r}$ may approach or exceed unity as $\mathbf{r}$ is very large. This may lead to additional energy loss intensity in our spectra, and cause the dipole transitions to be partially hidden by a "background" of nondipole transitions. However, we observe no obvious effects indicating a breakdown of the dipole selection rule, except possibly a general broadening of the features.

Based on the good agreement between the experimental and theoretical spectra, we wish to compare the theoretical dielectric function with the dielectric function derived directly from the experiments. The complex dielectric function of a material describes the electrical response of the material to external fields, and is usually obtained from optical experiments, but can also be retrieved from the low energy-loss spectra through deconvolution and subsequent analysis using the Kramers-Kronig relation. ${ }^{43}$ This approach has several advantages over the conventional optical methods: the experiments can be performed on minute amounts of nontransparent material and may yield the dielectric function over a larger range of frequencies. 
The Kramers-Kronig analysis successfully extracted the real and imaginary parts of the dielectric function from our experimental data from $\mathrm{CoAs}_{3}$, but was less successful for the other two materials. The experimental dielectric function of the As-based skutterudite is shown in Fig. 6 together with the theoretical dielectric function. Both the real and imaginary parts of the experimental dielectric function display peaks in the lower parts of the spectrum. These occur at approximately 1.8 and $2.4 \mathrm{eV}$, respectively, which is in excellent agreement with the DFT calculations that indicate maxima at 1.4 and $2.5 \mathrm{eV}$. The real part of the experimental dielectric function crosses zero at about $6.1 \mathrm{eV}$, which is significantly higher than the DFT value of $2.9 \mathrm{eV}$. There is also a significant difference in the slope of both the real and imaginary parts in the $4-10 \mathrm{eV}$ region. Despite these differences the overall agreement between experiment and theory is reasonable, suggesting that using EELS to investigate the dielectric function and comparing to theoretical calculations may be an important tool in studying the electronic structure of novel materials.

\section{CONCLUSION}

We achieve a reasonable agreement between the DFT calculated and EELS extracted dielectric functions in our study of binary skutterudites. However, there are also significant differences. The cause of these differences is not likely to be that our experiments are performed materials that deviate from the nominal compositions, nor defects in the material that disturbs the measurements. When it comes to details of the dielectric function there may be inaccuracies on the experimental side in the procedures used to correct the spectra for multiple scattering. However, EELS represents a highly robust and accurate method to measure the plasmon energy. Thus, the significant discrepancy between EELS measured and DFT calculated plasmon energies may inspire to further improvements of first-principle calculations. An interesting characteristic of EELS is that the technique can be used on nanometer sized volumes. Thus, plasmon energy measurements by EELS may become a valuable technique to test DFT calculations on nanomaterials, a parallel to use calorimetric data to test DFT calculations on bulk materials. ${ }^{52}$
${ }^{1}$ R. D. Leapman, L. A. Grunes, and P. L. Fejes, Phys. Rev. B 26, 614 (1982).

${ }^{2}$ R. D. Leapman and L. A. Grunes, Phys. Rev. Lett. 45, 397 (1980).

${ }^{3}$ T. I. Morrison, M. B. Brodsky, N. J. Zaluzec, and L. R. Sill, Phys. Rev. B 32, 3107 (1985).

${ }^{4}$ D. H. Pearson, C. C. Ahn, and B. Fultz, Phys. Rev. B 47, 8471 (1993).

${ }^{5}$ D. H. Pearson, C. C. Ahn, and B. Fultz, Phys. Rev. B 50, 12969 (1994).

${ }^{6}$ J. Graetz, C. C. Ahn, H. Ouyang, P. Rez, and B. Fultz, Phys. Rev. B 69, 235103 (2004).

${ }^{7}$ H. Ouyang and K. Jen-Tai, J. Appl. Phys. 92, 7510 (2002).

${ }^{8} \mathrm{H}$. Raether, Excitation of Plasmons and Interband Transitions by Electrons, Vol. 88 of Springer Tracts in Modern Physics (Springer-Verlag, Berlin, 1980).

${ }^{9}$ A. M. Sanches, R. Beanland, A. J. Papworth, G. J. Goodhew, and M. H. Grass, Appl. Phys. Lett. 88, 051917 (2006).

${ }^{10}$ T. Galek, C. Hérbert, D. Eyidi, T. Moskalewicz, and P. Schattschneider, J. Phys.: Condens. Matter 17, 3657 (2005).

${ }^{11}$ G. Oneida, L. Reining, and A. Rubio, Rev. Mod. Phys. 74, 601 (2002).

${ }^{12}$ O. Pulci, M. Marsili, E. Luppi, C. Hogan, V. Garbuio, F. Sottile, R. Magri, and R. D. Sole, Phys. Status Solidi B 242, 2737 (2005).

${ }^{13} \mathrm{~F}$. Jensen, Introduction to Computational Chemistry (Wiley, Chichester, England, 1999), Chap. 4.

${ }^{14}$ L. Hedin, J. Phys.: Condens. Matter 11, R489 (1999).

${ }^{15}$ M. Rohlfing and S. G. Louie, Phys. Rev. B 62, 4927 (2000).

${ }^{16}$ E. K. U. Gross, C. A. Ullright, and U. J. Grossmann, Density Functional Theory (Plenum Press, New York, 1994), p. 149.

${ }^{17}$ R. van Leeuwen, Int. J. Mod. Phys. B 15, 1969 (2001).

${ }^{18}$ K. Burke, M. Petersilka, and E. K. U. Gross, Recent Advances in Density Functional Methods (World Scientific, Singapore,
2002), Vol. III.

${ }^{19}$ F. Bruneval, F. Sottile, V. Olevano, and L. Reining, J. Chem. Phys. 124, 144113 (2006).

${ }^{20}$ E. J. Baerends and O. V. Gritsenko, J. Chem. Phys. 123, 062202 (2005).

${ }^{21}$ B. Walker, A. M. Saitta, R. Gebauer, and S. Baroni, Phys. Rev. Lett. 96, 113001 (2006).

${ }^{22}$ K. Karlsson, J. Phys.: Condens. Matter 17, 7573 (2005).

${ }^{23}$ L. Reining, V. Olevano, A. Rubio, and G. Onida, Phys. Rev. Lett. 88, 066404 (2002).

${ }^{24}$ P. Rez, J. R. Alvarez, and C. Pickard, Ultramicroscopy 78, 175 (1999).

${ }^{25}$ C. Hérbert, J. Luitz, and P. Schattschneider, Micron 34, 219 (2003).

${ }^{26}$ K. Lie, R. Høier, and R. Brydson, Phys. Rev. B 61, 1786 (2000).

${ }^{27}$ K. Lie, R. Brydson, and H. Davock, Phys. Rev. B 59, 5361 (1999).

${ }^{28}$ V. J. Keast, J. Electron Spectrosc. Relat. Phenom. 143, 97 (2005).

${ }^{29}$ P. Moreau and M. C. Cheynet, Ultramicroscopy 94, 293 (2003).

${ }^{30}$ V. Olevano and L. Reining, Phys. Rev. Lett. 86, 5962 (2001).

${ }^{31}$ C. Uher, Semicond. Semimetals 69, 139 (2001).

${ }^{32}$ M. Llunell, P. Alemany, S. Alvarez, V. P. Zhukov, and A. Vernes, Phys. Rev. B 53, 10605 (1996).

${ }^{33}$ D. J. Singh and W. E. Pickett, Phys. Rev. B 50, 11235 (1994).

${ }^{34}$ M. Fornari and D. J. Singh, Phys. Rev. B 59, 9722 (1999).

${ }^{35}$ K. Koga, K. Akai, K. Oshiro, and M. Matsuura, Phys. Rev. B 71, 155119 (2005)

${ }^{36}$ H. Anno, K. Matsubara, T. Caillat, and J. P. Fleurial, Phys. Rev. B 62, 10737 (2000).

${ }^{37}$ I. Lefebvre-Devos, M. Lassalle, X. Wallart, J. Olivier-Fourcade, L. Monconduit, and J. C. Jumas, Phys. Rev. B 63, 125110 (2001).

${ }^{38}$ A. P. Grosvenor, R. G. Cavell, and A. Mar, Chem. Mater. 18, 1650 (2006). 
${ }^{39}$ A. C. Larson and R. B. von Drele (unpublished).

${ }^{40}$ W. Jeitschko, A. J. Foecker, D. Paschke, M. V. Dewalsky, C. B. H. Evers, B. Kuennen, A. Lang, G. Kotzyba, U. C. Rodenwald, and M. H. Moeller, Z. Anorg. Allg. Chem. 626, 1112 (2000).

${ }^{41}$ N. Mandel and J. Donohue, Acta Crystallogr., Sect. B: Struct. Crystallogr. Cryst. Chem. 27, 2288 (1971).

${ }^{42}$ T. Schmidt, G. Kliche, and H. D. Lutz, Acta Crystallogr., Sect. C: Cryst. Struct. Commun. 43, 1678 (1987).

${ }^{43}$ R. F. Egerton, Electron Energy Loss Spectroscopy in the Electron Microscope, 1st ed. (Plenum Press, New York, 1986).

${ }^{44}$ G. Kresse and J. Hafner, Phys. Rev. B 47, R558 (1993).

${ }^{45}$ G. Kresse and J. Furthmüller, Phys. Rev. B 54, 11169 (1996).

${ }^{46}$ P. Blaha, K. Schwarz, G. Madsen, D. Kvasnicka, and J. Luitz,
WIEN2k, an Augmented Plane Wave + Local Orbitals Program for Calculating Crystal Properties (Karlheinz Schwarz, TU Wien, Austria, 2001).

${ }^{47}$ O. M. Løvvik and Ø. Prytz, Phys. Rev. B 70, 195119 (2004).

${ }^{48}$ C. C. Ahn and O. L. Krivanek, EELS Atlas (Gatan, Inc., Warrendale, USA, 1983).

${ }^{49}$ L. Hedin, Phys. Rev. 139, A796 (1965).

${ }^{50}$ C. C. Ahn, ed., Transmission Electron Energy Loss Spectrometry in Materials Science and the EELS Atlas, 2nd ed. (Wiley, Weinheim, 2004).

${ }^{51}$ S. Schamm and G. Zanchi, Ultramicroscopy 96, 559 (2003).

${ }^{52}$ R. E. Watson, M. Weinert, and M. Alatalo, Phys. Rev. B 65, 014103 (2002). 